\title{
An Optimal Control Problem Governed by Nonlinear First Order Dynamic Equation on Time Scales
}

\author{
Jian-Ping Sun $\mathbb{D}^{1},{ }^{1,2}$ Qiu-Yan Ren, ${ }^{1,3}$ and Ya-Hong Zhao ${ }^{2}$ \\ ${ }^{1}$ College of Electrical and Information Engineering, Lanzhou University of Technology, Lanzhou 730050, China \\ ${ }^{2}$ Department of Applied Mathematics, Lanzhou University of Technology, Lanzhou 730050, China \\ ${ }^{3}$ College of Technology and Engineering, Lanzhou University of Technology, Lanzhou 730050, China \\ Correspondence should be addressed to Jian-Ping Sun; jpsun@lut.cn
}

Received 20 March 2020; Revised 10 May 2020; Accepted 11 May 2020; Published 26 May 2020

Guest Editor: Rongwei Guo

Copyright (c) 2020 Jian-Ping Sun et al. This is an open access article distributed under the Creative Commons Attribution License, which permits unrestricted use, distribution, and reproduction in any medium, provided the original work is properly cited.

In this paper, we are concerned with a class of optimal control problem governed by nonlinear first order dynamic equation on time scales. By imposing some suitable conditions on the related functions, for any given control policy, we first obtain the existence of a unique solution for the nonlinear controlled system. Then, we study the existence of an optimal solution for the optimal control problem.

\section{Introduction}

The theory of time scales was introduced by Hilger in [1] in order to unify discrete and continuous analysis. Some foundational definitions and results from the calculus on time scales will be defined in Section 2. For more details, one can see [2-4].

In recent years, the calculus of variations and optimal control problems on time scales have attracted the attention of some researchers. For example, [5-8] discussed the calculus of variations on time scales and [9-12] studied some maximum principles on time scales, while [13-16] investigated the existence of optimal solutions or the necessary conditions of optimality for some optimal control problems on time scales.

In 2017, Guo [17] studied the projective synchronization problem of a class of chaotic systems in arbitrary dimensions. Firstly, a necessary and sufficient condition for the existence of the projective synchronization problem was presented. Secondly, an algorithm was proposed to obtain all the solutions of the projective synchronization problem. Thirdly, a simple and physically implementable controller was designed to ensure the realization of the projective synchronization. Finally, some numerical examples were provided to verify the effectiveness and the validity of the proposed results.
In 2020, Xu and Zhang [18] investigated general mean-field linear-quadratic (LQ) games of stochastic large-population system, where the individual diffusion coefficient could depend on both the state and the control of the agent, and the control weight in the cost functional could be indefinite. The asymptotic suboptimality property of the decentralized strategies for the LQ games was derived through the consistency condition. A pricing problem was also studied, for which the decentralized suboptimal price was obtained.

Throughout this paper, we always assume that $\mathbb{T}$ is a time scale, $T>0$ is fixed, $0, T \in \mathbb{T}$ and $\sigma^{2}(T)=\sigma(T)$. For each interval $\mathbf{I}$ of $\mathbb{R}$, we denote by $\mathbf{I}_{\mathbb{T}}=\mathbf{I} \cap \mathbb{T}$.

Suppose that there is a flock of sheep in a pasture. We consider the changes in the number of sheep during a time interval $[0, \sigma(T)]_{\mathbb{T}}$. It is well known that the supply of herbage, which influences growth rate and reproductive ability of sheep, is one of the main ways to control the number of sheep. Now, we define some related functions as follows:

$x(t)$ is the number of sheep at time $t$

$r(t)$ is the number of births per unit of time at time $t$ $p(t)$ is the number of sales per sheep per unit of time at time $t$ 
$u(t)$ is the amount of herbage supplied at time $t$

$q(t)$ is the number of sheep converted by per unit of herbage supplied per unit of time at time $t$

Let $U_{\text {ad }}$ be the admissible control set. Then, for any given control policy $u \in U_{\text {ad }}$, it is easy to know that the changes in the number of sheep can be described by the following linear dynamic equation:

$$
x^{\Delta}(t)+p(t) x(\sigma(t))=r(t)+q(t) u(t), \quad t \in[0, T]_{\mathbb{T}} .
$$

At the same time, in order to keep steady development, we may assume that the number of sheep at the beginning is equal to that at the end, that is,

$$
x(0)=x(\sigma(T)) .
$$

Suppose that $x_{u}$ is the solution of the controlled systems (1) and (2) corresponding to the control policy $u$ and $x_{d}$ is the desired value. Recently, the authors [19] considered the optimal control problem $\left(P_{0}\right)$. Find a $u_{0} \in U_{\text {ad }}$ such that

$$
J\left(u_{0}\right) \leq J(u), \quad \text { for all } u \in U_{\mathrm{ad}},
$$

where

$$
J(u)=\int_{0}^{T}\left[x_{u}(\sigma(t))-x_{d}(t)\right]^{2} \Delta t+\int_{0}^{T} u^{2}(t) \Delta t, \quad u \in U_{\mathrm{ad}},
$$

is the quadratic cost functional.

Motivated greatly by the abovementioned works, in this paper, we suppose that the controlled system is governed by the following more general nonlinear periodic boundary value problem:

$$
\left\{\begin{array}{l}
x^{\Delta}(t)+p(t) x(\sigma(t))=f(t, x(t), x(\sigma(t)))+g(u(t)), \quad t \in[0, T]_{\mathbb{T}} \\
x(0)=x(\sigma(T)) .
\end{array}\right.
$$

First, by imposing some suitable conditions on $p, f$, and $g$, for any given control policy $u \in U_{\text {ad }}$, we obtain the existence of a unique solution $x_{u}$ for the nonlinear controlled system (5). Then, we study the optimal control problem $(P)$. Find a $u_{0} \in U_{\text {ad }}$ such that

$$
J\left(u_{0}\right) \leq J(u), \quad \text { for all } u \in U_{\text {ad }},
$$

where

$$
J(u)=\int_{0}^{T}\left[x_{u}(\sigma(t))-x_{d}(t)\right]^{2} \Delta t+\int_{0}^{T} h(u(t)) \Delta t, \quad u \in U_{\mathrm{ad}},
$$

where $x_{d}$ is the desired value and $h: \mathbb{R} \longrightarrow[0, \infty)$ is continuous.

\section{Preliminaries}

In this section, we will provide some foundational definitions and results from the calculus on time scales.

Definition 1. We define the forward jump operator $\sigma: \mathbb{T} \longrightarrow \mathbb{T}$ by

$$
\sigma(t):=\inf \{s \in \mathbb{T}: s>t\}, \quad \text { for all } t \in \mathbb{T},
$$

while the backward jump operator $\rho: \mathbb{T} \longrightarrow \mathbb{T}$ is defined by

$$
\rho(t):=\sup \{s \in \mathbb{T}: s<t\}, \quad \text { for all } t \in \mathbb{T} .
$$

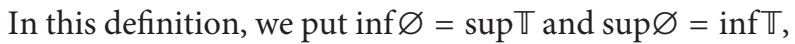
where $\varnothing$ denotes the empty set. If $\sigma(t)>t$, we say that $t$ is right-scattered, while if $\rho(t)<t$, we say that $t$ is left-scattered. Also, if $t<\sup \mathbb{T}$ and $\sigma(t)=t$, then $t$ is called right-dense, and if $t>\inf \mathbb{T}$ and $\rho(t)=t$, then $t$ is called left-dense. If $\mathbb{T}$ has a left-scattered maximum $m$, then we define $\mathbb{T}^{k}=\mathbb{T}-\{m\}$, otherwise $\mathbb{T}^{k}=\mathbb{T}$. Finally, the graininess function $\mu: \mathbb{T} \longrightarrow[0, \infty)$ is defined by

$$
\mu(t):=\sigma(t)-t, \quad \text { for all } t \in \mathbb{T} \text {. }
$$

Definition 2. Assume $f: \mathbb{T} \longrightarrow \mathbb{R}$ is a function and let $t \in \mathbb{T}^{k}$. Then, we define $f^{\Delta}(t)$ to be the number (provided it exists) with the property that given any $\varepsilon>0$, there is a neighborhood $U$ of $t$ (i.e., $U=(t-\delta, t+\delta)_{\mathbb{T}}$ for some $\left.\delta>0\right)$ such that

$$
\left|f(\sigma(t))-f(s)-f^{\Delta}(t)(\sigma(t)-s)\right| \leq \varepsilon|\sigma(t)-s|, \quad \text { for all } s \in U .
$$

We call $f^{\Delta}(t)$ the delta derivative of $f$ at $t$.

Moreover, we say that $f$ is delta differentiable (or in short, differentiable) on $\mathbb{T}^{k}$ provided $f^{\Delta}(t)$ exists for all $t \in \mathbb{T}^{k}$. The function $f^{\Delta}: \mathbb{T}^{k} \longrightarrow \mathbb{R}$ is then called the (delta) derivative of $f$ on $\mathbb{T}^{k}$. A function $F: \mathbb{T} \longrightarrow \mathbb{R}$ is called an antiderivative of $f: \mathbb{T} \longrightarrow \mathbb{R}$ provided

$$
F^{\Delta}(t)=f(t) \text { holds for all } t \in \mathbb{T}^{k} .
$$

If $F: \mathbb{T} \longrightarrow \mathbb{R}$ is an antiderivative of $f: \mathbb{T} \longrightarrow \mathbb{R}$, then we define the Cauchy integral by

$$
\int_{a}^{b} f(t) \Delta t=F(b)-F(a), \quad \text { for all } a, b \in \mathbb{T} .
$$

Definition 3. A function $f: \mathbb{T} \longrightarrow \mathbb{R}$ is called rd-continuous provided it is continuous at right-dense points in $\mathbb{T}$ and its left-sided limits exist (finite) at left-dense points in $\mathbb{T}$.

Definition 4 . We say that a function $p: \mathbb{T} \longrightarrow \mathbb{R}$ is regressive provided

$$
1+\mu(t) p(t) \neq 0, \quad \text { for all } t \in \mathbb{T}^{k},
$$

holds. The set of all regressive and rd-continuous functions will be denoted by $\mathscr{R}$. We define the set of positively 
regressive functions $\mathscr{R}^{+}$as the set consisting of those $p \in \mathscr{R}$ satisfying

$$
1+\mu(t) p(t)>0, \quad \text { for all } t \in \mathbb{T} \text {. }
$$

Lemma 1. Let $p \in \mathscr{R}, t_{0} \in \mathbb{T}$, and $e_{p}\left(\cdot, t_{0}\right)$ be the exponential function on $\mathbb{T}$. Then,

(i) $e_{p}(t, t) \equiv 1$ for all $t \in \mathbb{T}$

(ii) $e_{p}^{\Delta}\left(t, t_{0}\right)=p(t) e_{p}\left(t, t_{0}\right)$ for all $t \in \mathbb{T}^{k}$

Moreover, if $p \in \mathscr{R}^{+}$, then

$$
e_{p}\left(t, t_{0}\right)>0, \quad \text { for all } t \in \mathbb{T} \text {. }
$$

Lemma 2. Let $f$ be a continuous function on $[a, b]_{\mathbb{T}}$ that is differentiable on $[a, b)_{\mathbb{T}}$. Then, $f$ is increasing, decreasing, nondecreasing, and nonincreasing on $[a, b]_{\mathbb{T}}$ if $f^{\Delta}(t)>0$, $f^{\Delta}(t)<0, f^{\Delta}(t) \geq 0$, and $f^{\Delta}(t) \leq 0$ for all $t \in[a, b)_{\mathbb{T}}$, respectively.

Lemma 3. Let $\left\{f_{n}\right\}_{n=1}^{\infty}$ be a sequence of $\Delta$ integrable functions on $[a, b]_{\mathbb{T}}$ and suppose that $f_{n} \longrightarrow f$ uniformly on $[a, b)_{\mathbb{T}}$ for a function $f$ defined on $[a, b]_{\mathbb{T}}$. Then, $f$ is $\Delta$ integrable from a to $b$ and

$$
\int_{a}^{b} f(t) \Delta t=\lim _{n \longrightarrow \infty} \int_{a}^{b} f_{n}(t) \Delta t .
$$

In the remainder of this paper, we always assume that Banach space

$$
C\left([a, b]_{\mathbb{T}}, \mathbb{R}\right)=\left\{y \mid y:[a, b]_{\mathbb{T}} \longrightarrow \mathbb{R} \text { is continuous }\right\},
$$

is equipped with the norm $\|y\|=\max _{t \in[a, b]_{\pi}}|y(t)|$, $p:[0, T]_{\mathbb{T}} \longrightarrow(0, \infty)$ is rd-continuous, and denote

$$
M=\frac{1}{e_{p}(\sigma(T), 0)-1} .
$$

Then, it is easy to see that $M>0$.

Lemma 4 (see $[20])$. For any $y \in C\left([0, T]_{\mathbb{T}}, \mathbb{R}\right)$, the following first order linear periodic boundary value problem

$$
\left\{\begin{array}{l}
x^{\Delta}(t)+p(t) x(\sigma(t))=y(t), \quad t \in[0, T]_{\mathbb{V}}, \\
x(0)=x(\sigma(T)),
\end{array}\right.
$$

has a unique solution

$$
\begin{array}{r}
x(t)=\frac{1}{e_{p}(t, 0)}\left[\int_{0}^{t} e_{p}(s, 0) y(s) \Delta s+M \int_{0}^{\sigma(T)} e_{p}(s, 0) y(s) \Delta s\right], \\
t \in[0, \sigma(T)]_{\mathbb{V}} .
\end{array}
$$

\section{Main Results}

First, we list the following two conditions which we shall use in the sequel.

$\left(A_{1}\right) f:[0, T]_{\mathbb{T}} \times \mathbb{R}^{2} \longrightarrow \mathbb{R}$ is continuous and there exists $0<L<\left(M / 2(1+M)^{2} \sigma(T)\right)$ such that

$$
\begin{array}{r}
\left|f\left(t, \omega_{1}, v_{1}\right)-f\left(t, \omega_{2}, v_{2}\right)\right| \leq L\left(\left|\omega_{1}-\omega_{2}\right|+\left|v_{1}-v_{2}\right|\right), \\
t \in[0, T]_{\mathbb{T}}, \omega_{1}, \omega_{2}, v_{1}, v_{2} \in \mathbb{R} .
\end{array}
$$

$\left(A_{2}\right) g: \mathbb{R} \longrightarrow \mathbb{R}$ and there exists $K>0$ such that

$$
|g(\omega)-g(v)| \leq K|\omega-v|, \quad \omega, v \in \mathbb{R} .
$$

From now on, we always suppose that the control space is $C\left([0, T]_{\mathbb{T}}, \mathbb{R}\right)$ and the admissible control set $U_{\text {ad }}$ is a compact subset of $C\left([0, T]_{\mathbb{T}}, \mathbb{R}\right)$.

Lemma 5. Assume that conditions $\left(A_{1}\right)$ and $\left(A_{2}\right)$ are satisfied. Then, for any given control policy $u \in U_{a d}$, the nonlinear controlled system (5) has a unique solution $x_{u}$ and

$$
\begin{aligned}
x_{u}(t)= & \frac{1}{e_{p}(t, 0)}\left\{\int_{0}^{t} e_{p}(s, 0)\left[f\left(s, x_{u}(s), x_{u}(\sigma(s))\right)+g(u(s))\right] \Delta s\right. \\
& \left.+M \int_{0}^{\sigma(T)} e_{p}(s, 0)\left[f\left(s, x_{u}(s), x_{u}(\sigma(s))\right)+g(u(s))\right] \Delta s\right\}, \quad t \in[0, \sigma(T)]_{\mathbb{T}} .
\end{aligned}
$$

Proof. For any fixed $u \in U_{\text {ad }}$, we define an operator $\Phi_{u}: C\left([0, \sigma(T)]_{\mathbb{V}}, \mathbb{R}\right) \longrightarrow C\left([0, \sigma(T)]_{\mathbb{V}}, \mathbb{R}\right)$ as follows:

$$
\begin{aligned}
\left(\Phi_{u} x\right)(t)= & \frac{1}{e_{p}(t, 0)}\left\{\int_{0}^{t} e_{p}(s, 0)[f(s, x(s), x(\sigma(s)))+g(u(s))] \Delta s\right. \\
& \left.+M \int_{0}^{\sigma(T)} e_{p}(s, 0)[f(s, x(s), x(\sigma(s)))+g(u(s))] \Delta s\right\}, \quad t \in[0, \sigma(T)]_{\mathbb{T}} .
\end{aligned}
$$


Obviously, $x$ is a solution of the nonlinear controlled system (5) if and only if $x$ is a fixed point of $\Phi_{u}$ in $C\left([0, \sigma(T)]_{\mathbb{T}}, \mathbb{R}\right)$.
Let $x, y \in C\left([0, \sigma(T)]_{\mathbb{T}}, \mathbb{R}\right)$. Then, in view of Lemmas 1 and 2 and $\left(A_{1}\right)$, we have

$$
\begin{aligned}
\left|\left(\Phi_{u} x\right)(t)-\left(\Phi_{u} y\right)(t)\right|= & \frac{1}{e_{p}(t, 0)} \mid \int_{0}^{t} e_{p}(s, 0)[f(s, x(s), x(\sigma(s)))-f(s, y(s), y(\sigma(s)))] \Delta s \\
& +M \int_{0}^{\sigma(T)} e_{p}(s, 0)[f(s, x(s), x(\sigma(s)))-f(s, y(s), y(\sigma(s)))] \Delta s \mid \\
\leq & \frac{1}{e_{p}(t, 0)}\left\{\int_{0}^{t} e_{p}(s, 0)|f(s, x(s), x(\sigma(s)))-f(s, y(s), y(\sigma(s)))| \Delta s\right. \\
& \left.+M \int_{0}^{\sigma(T)} e_{p}(s, 0)|f(s, x(s), x(\sigma(s)))-f(s, y(s), y(\sigma(s)))| \Delta s\right\} \\
\leq & (1+M) \int_{0}^{\sigma(T)} e_{p}(s, 0)|f(s, x(s), x(\sigma(s)))-f(s, y(s), y(\sigma(s)))| \Delta s \\
\leq & L(1+M) \int_{0}^{\sigma(T)} e_{p}(s, 0)[|x(s)-y(s)|+|x(\sigma(s))-y(\sigma(s))|] \Delta s \\
\leq & 2 L(1+M)\|x-y\| \int_{0}^{\sigma(T)} e_{p}(s, 0) \Delta s \\
\leq & \frac{2 L(1+M)^{2} \sigma(T)}{M}\|x-y\|, \quad t \in[0, \sigma(T)]_{\mathbb{T}},
\end{aligned}
$$

so

$$
\left\|\Phi_{u} x-\Phi_{u} y\right\| \leq \frac{2 L(1+M)^{2} \sigma(T)}{M}\|x-y\|,
$$

which together with $0<L<\left(M / 2(1+M)^{2} \sigma(T)\right)$ implies that $\Phi_{u}: C\left([0, \sigma(T)]_{\mathbb{T}}, \mathbb{R}\right) \longrightarrow C\left([0, \sigma(T)]_{\mathbb{T}}, \mathbb{R}\right)$ is a contraction mapping.
Therefore, it follows from Banach contraction principle that $\Phi_{u}$ has a unique fixed point $x_{u} \in C\left([0, \sigma(T)]_{\mathbb{V}}, \mathbb{R}\right)$. This indicates that the nonlinear controlled system (5) has a unique solution $x_{u}$ and

$$
\begin{aligned}
x_{u}(t)= & \frac{1}{e_{p}(t, 0)}\left\{\int_{0}^{t} e_{p}(s, 0)\left[f\left(s, x_{u}(s), x_{u}(\sigma(s))\right)+g(u(s))\right] \Delta s\right. \\
& \left.+M \int_{0}^{\sigma(T)} e_{p}(s, 0)\left[f\left(s, x_{u}(s), x_{u}(\sigma(s))\right)+g(u(s))\right] \Delta s\right\}, \quad t \in[0, \sigma(T)]_{\mathbb{V}} .
\end{aligned}
$$

Theorem 1. Assume that conditions $\left(A_{1}\right)$ and $\left(A_{2}\right)$ are satisfied and $h: \mathbb{R} \longrightarrow[0, \infty)$ is continuous. Then, the optimal control problem $(P)$ has an optimal solution $u_{0} \in U_{a d}$.
Proof. First, it follows from Lemma 5 that, for any given control policy $u \in U_{\text {ad }}$, the nonlinear controlled system (5) has a unique solution $x_{u}$ and

$$
\begin{aligned}
x_{u}(t)= & \frac{1}{e_{p}(t, 0)}\left\{\int_{0}^{t} e_{p}(s, 0)\left[f\left(s, x_{u}(s), x_{u}(\sigma(s))\right)+g(u(s))\right] \Delta s\right. \\
& \left.+M \int_{0}^{\sigma(T)} e_{p}(s, 0)\left[f\left(s, x_{u}(s), x_{u}(\sigma(s))\right)+g(u(s))\right] \Delta s\right\}, \quad t \in[0, \sigma(T)]_{\mathbb{T}} .
\end{aligned}
$$


Next, in view of $J(u)=\int_{0}^{T}\left[x_{u}(\sigma(t))-x_{d}(t)\right]^{2} \Delta t+$ $\int_{0}^{T} h(u(t)) \Delta t, \quad u \in U_{\mathrm{ad}}$, it is obvious that $\inf _{u \in U_{\mathrm{ad}}} J(u)$ exists. Thus, by the definition of infimum, we know that there exists a sequence $\left\{u_{n}\right\}_{n=1}^{\infty} \subset U_{\text {ad }}$ such that

$$
\lim _{n \longrightarrow \infty} J\left(u_{n}\right)=\inf _{u \in U_{\mathrm{ad}}} J(u) .
$$

On the one hand, since $U_{\text {ad }}$ is a compact subset of $C\left([0, T]_{\mathbb{T}}, \mathbb{R}\right)$ and $\left\{u_{n}\right\}_{n=1}^{\infty} \subset U_{\text {ad }},\left\{u_{n}\right\}_{n=1}^{\infty}$ has a convergent subsequence in $U_{\text {ad }}$. Without loss of generality, we may assume that $\left\{u_{n}\right\}_{n=1}^{\infty}$ converges in $U_{\mathrm{ad}}$, that is, there exists $u_{0} \in U_{\text {ad }}$ such that

$$
\lim _{n \longrightarrow \infty} u_{n}=u_{0} .
$$

On the other hand, in view of Lemmas 1 and 2, $\left(A_{1}\right)$ and $\left(A_{2}\right)$, for any $n=1,2, \ldots$, we have

$$
\begin{aligned}
\left|x_{u_{n}}(t)-x_{u_{0}}(t)\right|= & \frac{1}{e_{p}(t, 0)} \mid \int_{0}^{t} e_{p}(s, 0)\left[f\left(s, x_{u_{n}}(s), x_{u_{n}}(\sigma(s))\right)-f\left(s, x_{u_{0}}(s), x_{u_{0}}(\sigma(s))\right)+g\left(u_{n}(s)\right)-g\left(u_{0}(s)\right)\right] \Delta s \\
& +M \int_{0}^{\sigma(T)} e_{p}(s, 0)\left[f\left(s, x_{u_{n}}(s), x_{u_{n}}(\sigma(s))\right)-f\left(s, x_{u_{0}}(s), x_{u_{0}}(\sigma(s))\right)+g\left(u_{n}(s)\right)-g\left(u_{0}(s)\right)\right] \Delta s \mid \\
\leq & \int_{0}^{t} e_{p}(s, 0)\left|f\left(s, x_{u_{n}}(s), x_{u_{n}}(\sigma(s))\right)-f\left(s, x_{u_{0}}(s), x_{u_{0}}(\sigma(s))\right)+g\left(u_{n}(s)\right)-g\left(u_{0}(s)\right)\right| \Delta s \\
& +M \int_{0}^{\sigma(T)} e_{p}(s, 0)\left|f\left(s, x_{u_{n}}(s), x_{u_{n}}(\sigma(s))\right)-f\left(s, x_{u_{0}}(s), x_{u_{0}}(\sigma(s))\right)+g\left(u_{n}(s)\right)-g\left(u_{0}(s)\right)\right| \Delta s \\
\leq & (1+M) \int_{0}^{\sigma(T)} e_{p}(s, 0)\left|f\left(s, x_{u_{n}}(s), x_{u_{n}}(\sigma(s))\right)-f\left(s, x_{u_{0}}(s), x_{u_{0}}(\sigma(s))\right)+g\left(u_{n}(s)\right)-g\left(u_{0}(s)\right)\right| \Delta s \\
\leq & (1+M) \int_{0}^{\sigma(T)} e_{p}(s, 0)\left[\left|f\left(s, x_{u_{n}}(s), x_{u_{n}}(\sigma(s))\right)-f\left(s, x_{u_{0}}(s), x_{u_{0}}(\sigma(s))\right)\right|+\left|g\left(u_{n}(s)\right)-g\left(u_{0}(s)\right)\right|\right] \Delta s \\
\leq & (1+M) \int_{0}^{\sigma(T)} e_{p}(s, 0)\left\{L\left[\left|x_{u_{n}}(s)-x_{u_{0}}(s)\right|+\left|x_{u_{n}}(\sigma(s))-x_{u_{0}}(\sigma(s))\right|\right]+K\left|u_{n}(s)-u_{0}(s)\right|\right\} \Delta s \\
\leq & (1+M)\left(2 L\left\|x_{u_{n}}-x_{u_{0}}\right\|+K\left\|u_{n}-u_{0}\right\|\right) \int_{0}^{\sigma(T)} e_{p}(s, 0) \Delta s \\
\leq & \frac{2 L(1+M)^{2} \sigma(T)}{M}\left\|x_{u_{n}}-x_{u_{0}}\right\|+\frac{K(1+M)^{2} \sigma(T)}{M}\left\|u_{n}-u_{0}\right\|, \quad t \in[0, \sigma(T)]_{\mathbb{T}},
\end{aligned}
$$

$$
\lim _{n \longrightarrow \infty} x_{u_{n}}=x_{u_{0}} .
$$

so, for any $n=1,2, \ldots$, we obtain

$$
\left\|x_{u_{n}}-x_{u_{0}}\right\| \leq \frac{K(1+M)^{2} \sigma(T)}{M-2 L(1+M)^{2} \sigma(T)}\left\|u_{n}-u_{0}\right\|
$$

which together with (31) implies that

$$
\begin{aligned}
\lim _{n \longrightarrow \infty} J\left(u_{n}\right) & =\lim _{n \longrightarrow \infty}\left(\int_{0}^{T}\left[x_{u_{n}}(\sigma(t))-x_{d}(t)\right]^{2} \Delta t+\int_{0}^{T} h\left(u_{n}(t)\right) \Delta t\right) \\
& =\int_{0}^{T} \lim _{n \longrightarrow \infty}\left[x_{u_{n}}(\sigma(t))-x_{d}(t)\right]^{2} \Delta t+\int_{0}^{T} \lim _{n \longrightarrow \infty} h\left(u_{n}(t)\right) \Delta t \\
& =\int_{0}^{T}\left[x_{u_{0}}(\sigma(t))-x_{d}(t)\right]^{2} \Delta t+\int_{0}^{T} h\left(u_{0}(t)\right) \Delta t \\
& =J\left(u_{0}\right),
\end{aligned}
$$


which together with (30) indicates that

$$
J\left(u_{0}\right)=\inf _{u \in U_{\mathrm{ad}}} J(u) .
$$

Therefore, $J\left(u_{0}\right) \leq J(u)$ for all $u \in U_{\text {ad }}$. This shows that $u_{0}$ is an optimal solution of the optimal control problem $(P)$.
Example 1. Let $\mathbb{T}=[0,1] \cup[2,3]$. We suppose that the controlled system is governed by the following nonlinear periodic boundary value problem

$$
\left\{\begin{array}{l}
x^{\Delta}(t)+x(\sigma(t))=D t^{2}\left[x(t) \arctan x(t)-\frac{1}{2} \ln \left(1+x^{2}(t)\right)+\frac{\pi}{4} \sin ^{2} x(\sigma(t))\right]+u(t), \quad t \in[0,3]_{\mathbb{T}}, \\
x(0)=x(3),
\end{array}\right.
$$

where

$$
D=\frac{2\left(2 e^{2}-1\right)}{9 \pi\left[28 e^{4}-4 e^{2}+1\right]} .
$$

In view of $\mathbb{T}=[0,1] \cup[2,3], T=3$ and $p(t) \equiv 1$ for $t \in[0,3]_{\mathbb{T}}$, it is not difficult to obtain that

$$
M=\frac{1}{2 e^{2}-1}
$$

Since $\quad f(t, \omega, v)=D t^{2}\left[\omega \arctan \omega-(1 / 2) \ln \left(1+\omega^{2}\right)+\right.$ $\left.(\pi / 4) \sin ^{2} v\right]$ for $(t, \omega, v) \in[0,3]_{\mathbb{T}} \times \mathbb{R}^{2}$ and $g(\omega)=\omega$ for $\omega \in \mathbb{R}$, it is obvious that $f:[0,3]_{\mathbb{T}} \times \mathbb{R}^{2} \longrightarrow \mathbb{R}$ is continuous and $\left(A_{2}\right)$ is satisfied. Moreover, if we choose $L=(9 \pi D / 2)$, then $0<L<\left(M / 2(1+M)^{2} \sigma(T)\right)$ and it follows from Lagrange mean value theorem that

$$
\begin{aligned}
& \left|f\left(t, \omega_{1}, v_{1}\right)-f\left(t, \omega_{2}, v_{2}\right)\right| \\
& \quad=D t^{2}\left|\omega_{1} \arctan \omega_{1}-\frac{1}{2} \ln \left(1+\omega_{1}^{2}\right)+\frac{\pi}{4} \sin ^{2} v_{1}-\omega_{2} \arctan \omega_{2}+\frac{1}{2} \ln \left(1+\omega_{2}^{2}\right)-\frac{\pi}{4} \sin ^{2} v_{2}\right| \\
& \quad \leq 9 D\left[\left|\omega_{1} \arctan \omega_{1}-\frac{1}{2} \ln \left(1+\omega_{1}^{2}\right)-\omega_{2} \arctan \omega_{2}+\frac{1}{2} \ln \left(1+\omega_{2}^{2}\right)\right|+\frac{\pi}{4}\left|\sin ^{2} v_{1}-\sin ^{2} v_{2}\right|\right] \\
& \quad \leq \frac{9 \pi D}{2}\left(\left|\omega_{1}-\omega_{2}\right|+\left|v_{1}-v_{2}\right|\right) \\
& \quad=L\left(\left|\omega_{1}-\omega_{2}\right|+\left|v_{1}-v_{2}\right|\right), \quad t \in[0,3]_{\mathbb{T}}, \omega_{1}, \omega_{2}, v_{1}, v_{2} \in \mathbb{R} .
\end{aligned}
$$

This shows that $\left(A_{1}\right)$ is fulfilled.

For any given constant $N>0$, let $U_{\text {ad }}=\left\{u \in C\left([0,3]_{\mathbb{T}}\right.\right.$, $\mathbb{R}) \mid u(0)=0$ and $\left|u\left(t_{1}\right)-u\left(t_{2}\right)\right| \leq N\left|t_{1}-t_{2}\right|$ for all $t_{1}, t_{2} \in$ $\left.[0,3]_{\mathbb{T}}\right\}$. Then, it is easy to verify that $U_{\text {ad }}$ is a compact subset of $C\left([0,3]_{\mathbb{T}}, \mathbb{R}\right)$.

By Lemma 5, we know that, for any given control policy $u \in U_{\text {ad }}$, the nonlinear controlled system (37) has a unique solution $x_{u}$.

Now, we consider the optimal control problem $\left(P^{*}\right)$. Find a $u_{0} \in U_{\text {ad }}$ such that

$$
J\left(u_{0}\right) \leq J(u), \quad \text { for all } u \in U_{\text {ad }},
$$

where

$$
J(u)=\int_{0}^{3}\left[x_{u}(\sigma(t))-x_{d}(t)\right]^{2} \Delta t+\int_{0}^{3} u^{2}(t) \Delta t, \quad u \in U_{\mathrm{ad}},
$$

where $x_{d}$ is the desired value.
Since $h(\omega)=\omega^{2}$ for $\omega \in \mathbb{R}, h: \mathbb{R} \longrightarrow[0, \infty)$ is continuous, thus, all the conditions of Theorem 1 are satisfied. Therefore, it follows from Theorem 1 that the optimal control problem $\left(P^{*}\right)$ has an optimal solution $u_{0} \in U_{\text {ad }}$.

\section{Conclusions}

In this paper, we consider a class of optimal control problem governed by nonlinear first order dynamic equation on time scales. First, by imposing some suitable conditions on the related functions and applying Banach contraction principle, for any given control policy, we obtain the existence of a unique solution for the nonlinear controlled system. Next, we prove that the optimal control problem has an optimal solution in the admissible control set. Finally, an example is also given to illustrate the main result of this paper. 


\section{Data Availability}

No data were used to support this study.

\section{Conflicts of Interest}

The authors declare that there are no conflicts of interest regarding the publication of this paper.

\section{Acknowledgments}

This work was supported by the National Natural Science Foundation of China (Grant no. 11661049).

\section{References}

[1] S. Hilger, "Analysis on measure chains-a unified approach to continuous and discrete calculus," Results in Mathematics, vol. 18, no. 1-2, pp. 18-56, 1990.

[2] V. Lakshmikantham, S. Sivasundaram, and B. Kaymakcalan, Dynamic Systems on Measure Chains, Kluwer Academic Publishers, Boston, MA, USA, 1996.

[3] M. Bohner and A. Peterson, Dynamic Equations on Time Scales: An Introduction with Applications, Birkhäuser, Boston, MA, USA, 2001.

[4] M. Bohner and A. Peterson, Advances in Dynamic Equations on Time Scales, Birkhäuser, Boston, MA, USA, 2003.

[5] Z. Bartosiewicz, N. Martins, and D. F. M. Torres, "The second Euler-Lagrange equation of variational calculus on time scales," European Journal of Control, vol. 17, no. 1, pp. 9-18, 2011.

[6] R. A. C. Ferreira, A. B. Malinowska, and D. F. M. Torres, "Optimality conditions for the calculus of variations with higher-order delta derivatives," Applied Mathematics Letters, vol. 24, no. 1, pp. 87-92, 2011.

[7] R. Hilscher and V. Zeidan, "First order conditions for generalized variational problems over time scales," Computers and Mathematics with Applications, vol. 62, no. 9, pp. 34903503, 2011.

[8] A. B. Malinowska, N. Martins, and D. F. M. Torres, "Transversality conditions for infinite horizon variational problems on time scales," Optimization Letters, vol. 5, no. 1, pp. 41-53, 2011.

[9] P. Stehlik and B. Thompson, "Maximum principles for second order dynamic equations on time scales," Journal of Mathematical Analysis and Applications, vol. 331, no. 2, pp. 913-926, 2007.

[10] R. Hilscher and V. Zeidan, "Weak maximum principle and accessory problem for control problems on time scales," Nonlinear Analysis: Theory, Methods \& Applications, vol. 70, no. 9, pp. 3209-3226, 2009.

[11] S. Zhou and H. Li, "Maximum principles for dynamic equations on time scales and their applications," Journal of Applied Mathematics, vol. 2014, Article ID 434582, 6 pages, 2014.

[12] M. Bohner, K. Kenzhebaev, O. Lavrova, and O. Stanzhytskyi, "Pontryagin's maximum principle for dynamic systems on time scales," Journal of Difference Equations and Applications, vol. 23, no. 7, pp. 1161-1189, 2017.

[13] Y. Gong and X. Xiang, "A class of optimal control problems of systems governed by the first order linear dynamic equations on time scales," Journal of Industrial \& Management Optimization, vol. 5, no. 1, pp. 1-10, 2009.
[14] G. Liu, X. Xiang, and Y. Peng, "Nonlinear integro-differential equations and optimal control problems on time scales," Computers \& Mathematics with Applications, vol. 61, no. 2, pp. 155-169, 2011.

[15] D. A. Carlson, "The existence of optimal controls for problems defined on time scales," Journal of Optimization Theory and Applications, vol. 166, no. 2, pp. 351-376, 2015.

[16] O. E. Lavrova, "Conditions for the existence of optimal control for some classes of differential equations on time scales," Journal of Mathematical Sciences, vol. 222, no. 3, pp. 276-295, 2017.

[17] R. Guo, "Projective synchronization of a class of chaotic systems by dynamic feedback control method," Nonlinear Dynamics, vol. 90, no. 1, pp. 53-64, 2017.

[18] R. Xu and F. Zhang, " $\epsilon$-nash mean-field games for general linear-quadratic systems with applications," Automatica, vol. 114, Article ID 108835, 2020.

[19] Q.-Y. Ren, J.-P. Sun, and Y.-H. Zhao, "The existence of optimal solutions for an optimal control problem defined on time scales," Dynamic Systems and Applications, 2014, In press.

[20] J.-P. Sun and W.-T. Li, "Existence of solutions to nonlinear first-order PBVPs on time scales," Nonlinear Analysis: Theory, Methods \& Applications, vol. 67, no. 3, pp. 883-888, 2007. 